\title{
DESCRIPTIVE ANALYSIS OF QUALITY OF INFORMATION OF DRUGS ON ANDROID-BASED INDIAN ONLINE PHARMACY APPLICATIONS: A PRELIMINARY ANALYSIS
}

\author{
SHAIKH SANA PERVEEN ${ }^{1 *}$, SHWETA SURVE ${ }^{2}$, NERURKAR RP ${ }^{1}$
}

${ }^{1}$ Department of Pharmacology, Topiwala National Medical College, Mumbai, Maharashtra, India, ${ }^{2}$ Department of Pharmacology, Lokmanya Tilak Municipal Medical College and General Hospital, Mumbai, Maharashtra, India. Email: dr.sana811@gmail.com

Received: 10 February 2020, Revised and Accepted: 11 March 2020

\section{ABSTRACT}

Objectives: The objectives of the study were as follows: 1) To analyze the completeness of drug-related information of various Indian pharmacy online android applications and its readability. 2)To analyze the ease of availability of drugs and barriers to access them on these apps.

Methods: A total of five popular pharmacy android apps in India (1mg, Netmeds, MedPlus, Pharmeasy, and Medikova) were chosen to analyze the prescribing information (PI) regarding five drugs, metformin, atenolol, ranitidine, ibuprofen, and alprazolam. Display of PI in these apps was compared with standard sources, i.e., National Formulary of India, Current Index of Medical Specialties, and package inserts of respective drugs. Drug-related information under 11 categories (indication, dose, contraindications, adverse events, etc.) was analyzed in these apps as complete/ incomplete. Readability of information was assessed using Flesch Reading Ease Score (FRES). These five drugs were ordered from all the apps to know the exact process to access them.

Results: Categories such as indication and dose were mentioned in most apps. While, precautions and adverse events were given for few drugs in few apps. Least score was for categories like precautions in special population. FRES was minimum in Pharmeasy (30.82) and maximum in MedPlus (70.51). Valid prescriptions were demanded by all apps while one app had the facility of providing prescription from their own consultant. Only one app provided "Ask a Pharmacist" option.

Conclusion: We found lots of inconsistency in the information provided by these apps. Our results are based on only five apps and provide a framework for future studies to scrutinize online apps.

Keywords: Online pharmacy, Prescribing information, Flesch Reading Ease Score.

(C) 2020 The Authors. Published by Innovare Academic Sciences Pvt Ltd. This is an open access article under the CC BY license (http://creativecommons. org/licenses/by/4. 0/) DOI: http://dx.doi.org/10.22159/ajpcr.2020.v13i5.37099

\section{INTRODUCTION}

Online pharmacies are internet-based vendors that sell prescription as well as non-prescription medicines through online portals such as websites and mobile applications [1]. Retail pharmacies had thus far played a dominant role in the sale of medicines to the Indian population. However, with the advancement of technology, e-commerce emerged and opened another arena for vendors to market and sell their products [2]. Subsequent to this there was a boost in online shopping which became hassle free through the introduction of newer age smartphones. Online pharmacies have been successful in attracting a growing number of customers with their convenient home delivery services, $24 \times 7$ accessibility, lucrative deals, low transaction, and product cost, but such direct procurement of drugs could be hazardous as it would be difficult to determine whether the drugs are counterfeit, unapproved, or illegal [3].

The online sale of drugs started early in the year 2000 and has expanded so much that the US Food and Drug Administration (FDA) implemented an entire section on its website dedicated to "Buying medicines over the Internet" [4,5]. In India, there are no laws dedicated to regulate online pharmacies per se. The laws applicable for regulating the sales of drugs in India are the Drugs and Cosmetics Act, 1940; Drugs and Cosmetic Rules, 1945; Pharmacy Act, 1948; and the Indian Medical Act, 1956, which mention the guidelines on the sale of Schedule $\mathrm{H}$ and Schedule X medicines (restrictive medicines). These laws do not permit home delivery of drugs and drugs can be sold only on the prescription of a registered medical practitioner. Laws related to e-commerce are defined under the Information Technology Act, 2000 [6]. However, these laws are not well defined for online pharmacies and are subject to varied interpretations.

Online pharmacies work either through websites or through mobile applications available on android/iOS smartphones. Since these webbased portals lack patient-pharmacist communication, they become an important source of dissemination of prescribing information (PI) to consumers regarding the dosing schedule, route of administration, indications, adverse effects, contraindications, warnings, and precautions while taking the drugs [7]. The information thus provided should be scientifically accurate, complete, up to date, easy to understand, and useful. The availability of well-designed high-quality information on these pharmacy apps may improve patient safety, trust, and understanding of the information. While the accessibility of medicines through online pharmacies has been described, the quality of information disseminated through these apps has not been studied.

Thus, we carried out this pilot study to evaluate the quality of drugrelated information, its completeness, and understandability and to study the various barriers to access drugs in Indian online pharmacy android applications.

\section{Aims and objectives}

The objectives of the study were as follows:

1. To analyze the completeness of PI of various Indian pharmacy online android applications and its readability.

2. To analyze the ease of availability of drugs and barriers to access them on these apps. 


\section{MATERIALS AND METHODS}

\section{Selection of online android pharmacy apps}

We selected the five most popular online pharmacy android apps in India, delivering to Mumbai based on the greatest number of downloaded apps and the app rating they received on the android Play Store. The apps included 1mg, Netmeds, MedPlus, Pharmeasy, and Medikova.

\section{Analysis of content quality}

We chose five drugs that represent various therapeutic areas such as,

1. Antidiabetic: Metformin.

2. Antihypertensive: Atenolol.

3. Gastritis: Ranitidine.

4. Analgesic: Ibugesic

5. Antianxiety/sedative: Alprazolam.

PI of five popularly prescribed medications, metformin, atenolol, ranitidine, ibugesic, and alprazolam was analyzed [8]. PI included the indication, dose, precaution, adverse events, contraindications, drug-drug interactions, food-drug interactions, and its use in special populations such as pediatrics, geriatrics, and pregnancy. Display of PI in the apps was compared with standard sources, i.e., National Formulary of India, Current Index of Medical Specialties, and package inserts of respective drugs.

For every complete information, we gave a score of 1 , while incomplete information was given 0 . We evaluated each category from all five apps and gave a score out of 5. All scores were also expressed as a percentage.

\section{Readability assessment}

Information on these apps must have fewer medical terms and are easy to comprehend by consumers. Hence, we assessed the readability of each app using the Flesch Reading Ease Score (FRES). The Flesch readability score $(0-100)$ is based on the average number of syllables per word and words per sentence. Higher the score, easier it is to read the text. Although other readability instruments exist, we chose FRES due to the simplicity of calculating the readability score [9].
Ease of availability and barriers

We ordered all five drugs from these apps to see the availability of these drugs with or without a prescription and whether these prescriptions were validated by the apps.

We also checked if substitutes for the ordered drugs were available. Moreover, for the ease of finding substitutes, if "Ask a pharmacist," option was available.

All data were collected in July-August 2018.

\section{RESULTS}

The PI in different apps was analyzed by two investigators, who came to a consensus.

\section{Quality assessment}

The details of the content of quality of PI are given in Table 1.

\section{Indication}

It was mentioned in most apps for the above-mentioned medications except few, which were incomplete. $1 \mathrm{mg}$ and Netmeds did not give complete indications about alprazolam, but this drug is not sold online.

Dose

Correct dose was mentioned in all apps for all drugs except Netmeds which did not mention a dose of any drug.

\section{Precautions}

Precautions on taking medications like metformin were mentioned in two out of five apps. For atenolol, sudden withdrawal of the drug should be avoided which was mentioned in only one app, while for ranitidine, alprazolam, and ibugesic was given in three out of five apps.

\section{Adverse drug reactions}

ADRs for drugs such as metformin, atenolol, ranitidine, and alprazolam were given in two out of five apps. While for ibugesic, ADRs were given in three out of five apps.

Table 1: Completeness of PI

\begin{tabular}{|c|c|c|c|c|c|c|}
\hline \multicolumn{7}{|c|}{ PI in different categories for drugs (\%), n=5 } \\
\hline Categories & Metformin & Atenolol & Ranitidine & Alprazolam & Ibugesic & Total \\
\hline Indications & $4 / 5(80)$ & $4 / 5(80)$ & $3 / 5(60)$ & $3 / 5(60)$ & $3 / 5(60)$ & $17 / 25(68)$ \\
\hline Dose & $4 / 5(80)$ & $4 / 5(80)$ & $4 / 5(80)$ & $4 / 5(80)$ & $4 / 5(80)$ & $20 / 25(80)$ \\
\hline Precautions & $2 / 5(40)$ & $1 / 5(20)$ & $3 / 5(60)$ & $3 / 5(60)$ & $3 / 5(60)$ & $12 / 25(48)$ \\
\hline Adverse events & $2 / 5(40)$ & $2 / 5(40)$ & $2 / 5(40)$ & $2 / 5(40)$ & $3 / 5(60)$ & $11 / 25(44)$ \\
\hline Contraindications & $2 / 5(40)$ & $1 / 5(20)$ & $2 / 5(40)$ & $2 / 5(40)$ & $1 / 5(20)$ & $8 / 25(32)$ \\
\hline Drug-drug Interactions & $3 / 5(60)$ & $1 / 5(20)$ & $1 / 5(20)$ & $2 / 5(40)$ & $1 / 5(20)$ & $8 / 25(32)$ \\
\hline Food-drug interactions & $2 / 5(40)$ & $1 / 5(20)$ & $2 / 5(40)$ & $3 / 5(60)$ & $2 / 5(40)$ & $10 / 25(40)$ \\
\hline Pregnancy & $3 / 5(60)$ & $3 / 5(60)$ & $3 / 5(60)$ & $3 / 5(60)$ & $2 / 5(40)$ & $14 / 25(56)$ \\
\hline Pediatrics & $1 / 5(20)$ & $1 / 5(20)$ & $1 / 5(20)$ & $1 / 5(20)$ & $1 / 5(20)$ & $5 / 25(20)$ \\
\hline Geriatrics & $1 / 5(20)$ & $1 / 5(20)$ & $1 / 5(20)$ & $1 / 5(20)$ & $1 / 5(20)$ & $5 / 25(20)$ \\
\hline Reference & $1 / 5(20)$ & $1 / 5(20)$ & $1 / 5(20)$ & $1 / 5(20)$ & $1 / 5(20)$ & $5 / 25(20)$ \\
\hline
\end{tabular}

PI: Prescribing information, n: number of pharmacies

Table 2: FRES score for drugs

\begin{tabular}{llllll}
\hline Drug & $\mathbf{1 m g}$ & Netmeds & Pharmeasy & MedPlus & Medikova \\
\hline Metformin & 44.2 & 58.1 & 45.1 & 70.3 & 55 \\
Atenolol & 70 & 59.3 & 28.3 & 80 & 63 \\
Ranitidine & 59.6 & 48.7 & 46.4 & 63.8 & 55.8 \\
Alprazolam & 58.5 & 0 & 0 & 58.6 & 0 \\
Ibugesic & 49.7 & 63.3 & 34.3 & 80 & 48.2 \\
Mean score & $56.4 \pm 9.92$ & $45.88 \pm 26.20$ & $30.82 \pm 18.81$ & $70.51 \pm 9.58$ & $44.40 \pm 25.37$ \\
\hline
\end{tabular}

FRES: Flesch Reading Ease Score 


\section{Contraindications}

It was mentioned in two out of five apps for metformin, ranitidine, and alprazolam, while it was mentioned in only one app for atenolol and ibugesic.

\section{Drug-drug interactions}

It was mentioned in three apps for metformin, in two apps for alprazolam. It was mentioned in only one app for atenolol, ranitidine, and ibugesic.

\section{Food-drug interactions}

It mentioned in three apps for alprazolam, in two apps for metformin, ranitidine and ibugesic. Only one app had for atenolol.

Precautions in special population; pediatric and geriatric

It was mentioned in only one app.

Reference: The PI was mentioned in only one app.

None of the apps had a full score/complete information.

\section{Readability assessment}

Evaluation of readability by FRES on different apps ranged from 44 to 80. The average FRES score in apps was maximum in MedPlus, 70.51 (Fig. 1).

\section{Ease of availability and barriers}

We found all apps demanded prescription. Of these, four apps verified the prescription for its completeness.

None of the apps verified the prescription on delivery.

Only one app, i.e., Pharmeasy had an "Ask-a pharmacist" option.

\section{DISCUSSION}

Although the physician is supposed to provide all the important information of the prescribed drug, it is practically impossible. Hence, a pharmacy app may be helpful (added advantage) in providing such information. Furthermore, for chronic diseases such as hypertension and diabetes mellitus, patients do not visit the doctor regularly. Such patients are also likely to buy drugs online more often.

Indications for the drug use were given in most apps for most drugs evaluated; however, none of them provided all the indications as per our references. Similarly, doses were mentioned almost $80 \%$ of the time. Precautions, adverse drug reactions, and contraindications are crucial information associated with drug use. Although physicians usually keep this in mind while prescribing drugs, such information if available would be beneficial.

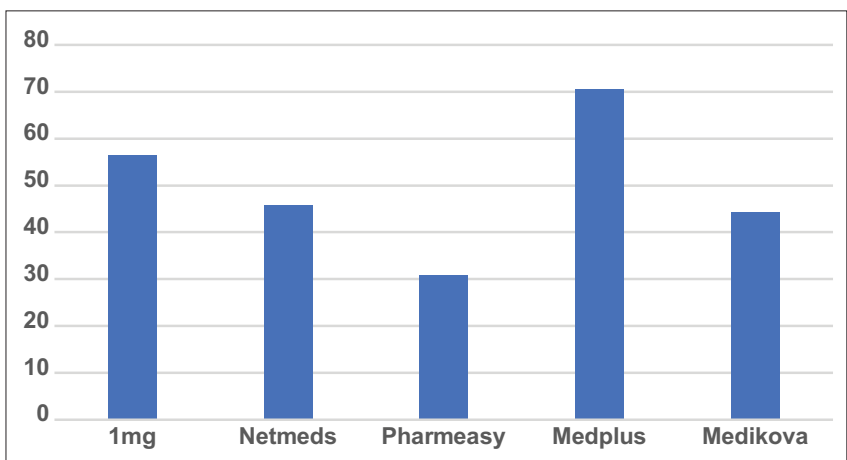

Fig. 1: Flesch Reading Ease Score (average) in apps for different drugs
One of the risks of online pharmacies is drug misuse, particularly with drugs having abuse potential. A prescription audit is necessary before dispensing such drugs. In our study, we found all apps demanded a prescription; however, only four apps verified the prescription for its completeness. Furthermore, none of the apps sold drugs with abuse potential like alprazolam. Orzio et al. conducted a similar study on online pharmacies and reported only a few websites requiring a valid prescription [10]. A systematic review by Grazia et al. reported that a few online websites did not ask for a prescription and replaced it with an online questionnaire [11].

We uploaded the same prescription on all five apps and observed that one prescription can be used to order the same drugs from multiple apps. This is alarming as the chances of unsupervised medications increase which is dangerous.

The option to get drug substitutes in case the patient is prescribed with the brand name was available with all apps. Although, this could create ambiguity if a sound-alike or look-alike drug is available. For this, Ask-apharmacist option is usually available. However, in our study, we found that only one app had "Ask-a pharmacist" option. We did not evaluate the quality of information provided by Ask-a-pharmacist option in this app. A study conducted by Holmes et.al. reported that a very poor response and inadequate information was provided by this option on various websites [12]. One app did not provide a generic name which makes it even more difficult to check for substitutes.

We found that readability scores were an average of 60 , which indicates that it is fairly difficult to read the information given on these apps. The information thus given would be worthless if the consumer is not able to interpret it. A study by Kunz et al. on readability levels of online pharmacy websites reported an average score of 55 [13].

\section{CONCLUSION}

Online pharmacies pose a considerable risk when it comes to buying/ selling of medications. With many deficiencies in the current regulations, it is essential to have stricter laws for the online sale of medications. Our study results are based on five drugs analyzed and purchased from five apps only; therefore, the results cannot be generalized beyond these. We aimed to screen these apps so as to provide a framework for future studies to scrutinize online apps.

\section{ACKNOWLEDGMENT}

This article was presented at Southern Regional Indian Pharmacological Society Conference, 2017, at Mahatma Gandhi Medical College and Research Institute, Pondicherry.

\section{AUTHORS' CONTRIBUTIONS}

Dr. S. P. Shaikh - Data collection, data analysis, and article writing. Dr. S. R. Surve - Data collection, data analysis, and article writing. Dr. R. P. Nerurkar - Article writing and final editing.

\section{REFERENCES}

1. Desai C. Online pharmacies: A boon or bane? Indian J Pharmacol 2016;48:615-6.

2. Alamelu R, Amudha R, Motha LC, Nalini R. Online pharma retail is a promising/unpromising avenue: An Indian context. Asian J Pharm Clin Res 2016;9:26-9.

3. Montoya ID, Jano E. Online pharmacies: Safety and regulatory considerations. Int J Health Serv 2007;37:279-89.

4. Gallagher JC, Colaizzi JL. Issues in Internet pharmacy practice. Ann Pharmacother 2000;34:1483-5.

5. US Food and Drug Administration US Department of Health and Human Services. Buying Medicines Over the Internet; 2019. Available from: http://www.fda.gov/drugs/resourcesforyou/consumers/ buyingusingmedicinesafely/buyingmedicinesovertheinternet/default. htm. [Last accessed on 2019 Dec 17].

6. Priyanka VP, Ashok BK. E-pharmacies regulation in India: Bringing 
new dimensions to pharma sector. Pharm Regul Aff 2016;5:1-7.

7. Weiss AM. Buying prescription drugs on the internet: Promises and pitfalls. Cleve Clin J Med 2006;73:282-8.

8. Yada NI, Thrulapati DT, Maheshwari AR, Upadhyay VI, Shah PR. A study on the prescribing pattern of antidiabetic drugs in a community clinic in Telangana state. Int J Pharm Pharm Sci 2015;7:222-6.

9. Flesch R. A new readability yardstick. J Appl psychol 1948;32:221-33

10. Orizio G, Schulz P, Domenighini S, Caimi L, Rosati C, Rubinelli S, et al. Cyberdrugs: A cross-sectional study of online pharmacies characteristics. Eur J Public Health 2009;19:375-7.

11. Orizio G, Merla A, Schulz PJ, Gelatti U. Quality of online pharmacies and websites selling prescription drugs: A systematic review. J Med Internet Res 2011;13:e74.

12. Holmes ER, Desselle SP, Nath DM, Markuss JJ. Ask the pharmacist: An analysis of online drug information services. Ann Pharmacother 2005;39:662-7.

13. Kunz MB, Osborne P. A preliminary examination of the readability of consumer pharmaceutical web pages. J Mark Dev Compet 2010;5:33-41. 